found. Fibrin is found about the veins and adjacent to the nerve tissue.

The arteries characteristically show lifting of the endothelium by cellular exudate. The veins often show. proliferative changes in the intima with infiltration by polynuclear leucocytes (characteristic in large sulcal veins). Two cases showed mural thrombus formation in the veins.

Seven cases out of twelve showed increase or other signs of change in the neuroglia, especially of the subpial layer.

Penetration of the cortical tissue by polynuclear leucocytes is almost constant.

Orbital inoculations in the guinea-pig showed remarkable variety in the results with different cultures. With the cultures yielding positive results, a general but not constant tendency is to the production of exudates with a high proportion of mononuclear cells of the phagocytic series. A series of orbital inoculations with culture identical throughout exhibited clearly the same tendency. The exudation of polynuclear leucocytes is primary, however, and may be noted in six hours. The exudate is at its height in three, four or five days and leaves no trace in from two to five weeks. Ependymitis and encephalitis are not prominent. The guinea-pig inoculations, as a rule, produce no clinical sign.

\section{THE DUMB-BELL INTESTINAL ANASTOMOSIS.}

A PRELIMINARY REPORT ON A NEW MECHANICAL DEVICI AND A NEW METHOD FOR ĖITHER INTESTINAL APPROXIMAATION OR ANASTOMOSIS WITH THE STOMACH.*

JOSEPH B. BACON, M.D.

Surgeon to the St. Francis Hospital. MACOMB, ILL.

The numerous methods of suturing that are described and the various mechanical devices that are demonstrated, even in our recent standard text-books of surgery, prove that the ideal method for intestinal approximation of anastomosis has not been discovered. Some of the methods of suturing have been successfully used by surgeons in a series of cases until they were almost ready to declare the method all that could be desired, when a sad accident occurred and the postmortem showed a defect in its workings. The same may be truthfully said of all the mechanical devices up to the present time.

The new method of operating and the new device here explained is the result of a year's work. I began with the idea that, in an end to end approximation of an intestine or an anastomosis of the intestine with the stomach; either where the severed end is used to make the anastomosis or with a lateral union, all of the connective tissues must be incorporated in the fixation method in order to safeguard against leakage, and also to get a firm safe union. This is what nature does in all cases in which one organ becomes agglutinated to another, and ultimately becomes anastomosed as a result of septic infection. After experimenting with one device after another, I finally succeeded in making this simple hollow dumb-bell accomplish the desired result. It simply acts as a hollow cylinder for the passage of liquids and gas, and at the same time gives a base on which we can tie all the connective tissue in one firm grasp by means of a rubber or silk suture of course fiber, and thus have a firm union for three or four days while the serosa and muscularis are becoming united by an organized lymph

* liead before the Chicago Academy of Medicine, 1905. in their new position. In from three to four days the organized tissue is a safe barrier against leakage. The ligature necroses through the connective tissue by this time and frees the device.

The dumb-bell is made of aluminum and is very light. There are three different sizes, one for children, one for adults and one for the colon. This same method may be used with a dumb-bell moulded from soft rubber or from any digestible material, but I prefer one of aluminum, as it can be made small, is light, has a definite strength so that one can do a definite work and know what to expect during a definite time.

We desire the connective tissue to hold at least for three days and prefer it to hold for five. My experiments on pigs proved that the dumb-bell is freed after from four to six days. The rubber ligature should be firmly wrapped around the tissues and the dumb-bell twice, then securely tied with several knots. The knots and ligatures are left within the canal and the quantity of ligature can make no difference. The ligature material should be coarse, so that it will necrose through slowly, hence use a rubber band about one-fourth inch broad (or heavy braided silk). Silk or linen sutures are not so safe as rubber in the hands of a beginner, as any sawing motion in applying the ligature might cut through and thus necessitate reamputation of the gut and a serious prolongation of the operation.

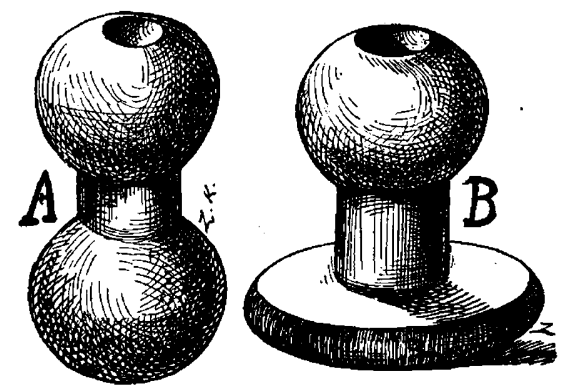

Fig. 1.-Showing the two styles of the dumb bells.

Operation for end to end approximation: The two ends of the bowel are secured by a suture at the mesenteric border, care being taken to include in this suture the V-shaped mesenteric triangles. Directly opposite the mesentery, on the periphery of the intestine, another suture is placed. Each of these sutures is tied loosely, as they are only used for the purpose of invaginating the two ends of the bowel. Two inches from the end of the intestine, along its peripheral border, an incision is made through the intestinal wall, one inch in length. This incision is made in the proximal end of the intestine, if it be enlarged, otherwise on the distal end. A pair of forceps is now passed through the incision and into the end of the intestine and the tension sutures secured and drawn through the incision. Now steadying the mesenteric border of the intestine between thumb and finger, and drawing the sutures, invaginates the two ends of the intestine into each other and through the incision. The dumb-bell is now placed into the ends of the invaginated intestine and the ligature applied at a distance of one-half inch from the ends of the severed intestine, encircling it and the handle of the dumb-bell. Disinvaginate by gentle traction and pressure on the dumbbell; reunite the peripheral incision by a Czerny-Lembert or Gèly suture. Then complete the operation by suturing the mesenteric opening.

The three sizes of the dumb-bell can by the ligature method be made to fulfill any requirement in any operation from the cardiac end of the stomach to the rectum. 
In doing a gastroduodenostomy an opening is made on the ventral wall of the stomach one and one-half inches in length. Through this aperture by means of a pair of forceps the dumb-bell is forced through the pyloric opening and dropped into the duodenum, where it can be pushed along by the hand within the abdomen to any selected point of the intestine, as the small omental cavity is opened for inspection, just as in any dorsal gastroenterostomy.

If there be a stenosis of the pylorus, an incision must be made in the duodenum near the pylorus and the dumb-bell placed in the duodenum, and this incision sutured. When the point is selected, with the right hand seize the dumb-bell from within the stomach with tenaculum forceps, then invaginate the duodenal wall and

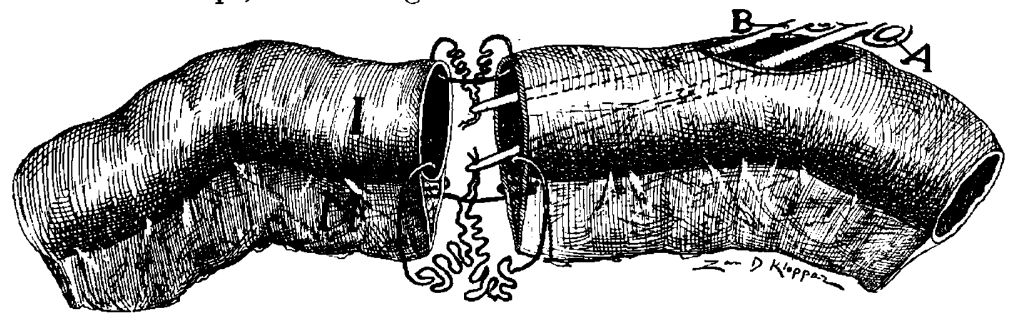

eration showed absolutely no leakage, the ligature and dumb-bell were firmly in place and a safe protective band of lymph was becoming organized. Especially at the dangerous mesenteric border can the accurate union be 'noticed. The strangulated ends of the intestine external to the ligature were very much hypertrophied, and dilated the intestine over the small end of the dumb-bell. For this reason $I$ have made the opposite end of the dumb-bell one-fourth inch larger, so as more accurately to coapt the surfaces during the first twenty-four hours.

A second specimen, taken forty-eight hours from the time of the operation, showed very firm organized lymph. The strangulated rim that was hypertrophied in the other specimen was receding here. The ligature, where the intestine was dissected, still held firmly and could not leak.

In a third specimen, taken from a pig six months after the operation, there was great difficulty in finding the place of union. The lumen was normal and there was but a bare trace of circular cicatrix. It could only be

Fig. 2.-Traction sutures grasped by forceps $A$ and $B$.

dorsal stomach wall through the former incision on the ventral stomach wall by pressing the ventral wall down over the dumbbell. Tie a firm ligature of heavy silk, reinforced by a rubber ligature, to insure uniform pressure, and necrosis.

Precaution must be used in not selecting a dumb-bell of more than one-half inch in

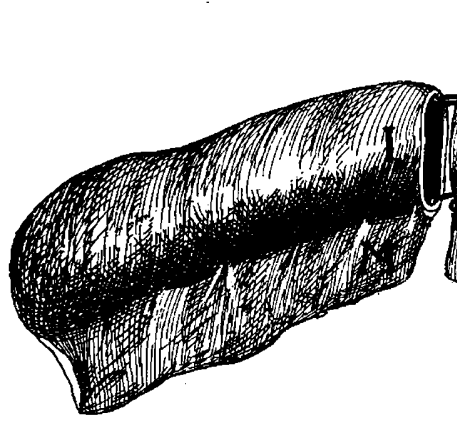

Fig. 3.-The traction sutures in place.

diameter, or excessive amount of the peripheral side of the small intestine will be ligated.

For uniting the severed end of the ileum to the site of the colon push the dumb-bell into the end of the intestine out of the way, throw a pursestring ligature around the end of the severed intestine, and leave the needle threaded. Select the point of the colon for anastomosis; nearly opposite this point open the colon by an inch incision, preferably in the tenia coli. Now insert the needle at the previous point selected and pull it out through this incision, and draw the side of the sigmoid and the end of the intestine into this incision. Now force the dumb-bell forward to the end of the intestine and place the ligature over the small end of the dumbbell. Suture the opening into the colon and also the mesentery of the small intestine.

Where the cecum has been amputated the work can be done through the end of the colon before it has been sutured. Experience has taught us that the small intestine should never be united end to end with the large intestine, but always lateral above a newly-made cecum.

A specimen removed twenty-four hours after the op-
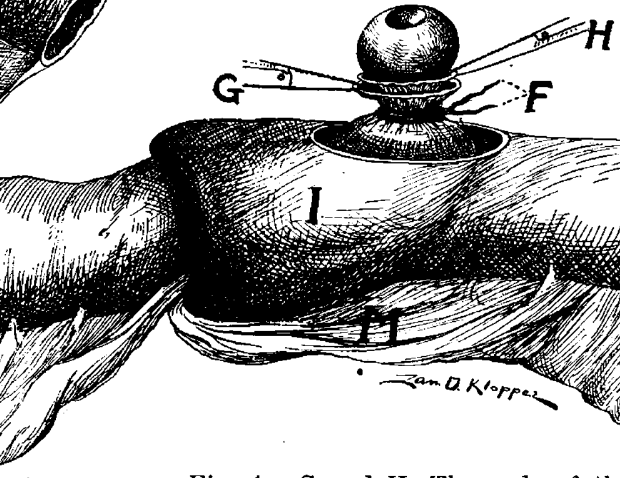

Fig. 4.-G and H. The ends of the invaginated intestine. F. Rubber ligature.

detected in a good light, and could barely be distinguished by touch. This pig weighed thirty-five pounds in May and 212 pounds when killed in November. The dumb-bell was evacuated on the tenth day.

A fourth specimen was secured from a pig

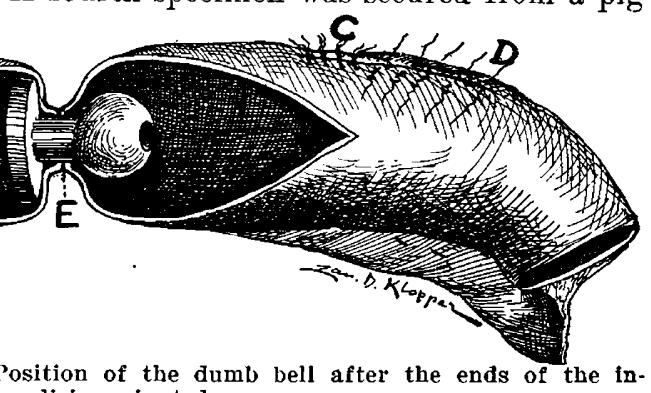

Fig. 5.-E. Position of the du
have been disinvaginated.

8 months after the operation. There was but a very slight limitation of the intestine. The cicatrix was barely to be distinguished. On inverting the specimen it showed a beautiful line of union of the mucous membrane. The union could barely be felt, and only showed a circular ring with a minimum amount of cicatricial tissue. This pig weighed fifty-four pounds in April at the time of the operation, and 340 pounds when killed in December. The dumb-bell was evacuated in this animal in eight days.

The advantages of this method of operating are:

1. Simplicity of the construction of the dumb-bell; therefore, always ready.

2. Simplicity of the operation.

3. The absolute safeguard against leakage, as the ligature secures all of the connective tissue. 
4. The short time in which the operation can be performed.

5. The dumb-bell cannot remain at the site of operation more than four or six days, as the connective tissue will all have been uniformly necrosed by that time.

6. The extremely light weight of the dumb-bell, its size and shape insure against the possibility of its lodging at any part of the alimentary tract.

$\%$. No reinforced sutures are necessary.

8. The minimum amount of cicatricial tissue that ultimately remains.

\section{ROENTGEN DIAGNOSIS OF DISEASES OF THE LUNGS.*}

\section{G. E. PFAHLER, M.D.}

Director of the Roentgen Ray Laboratory, and Assistant Physi cian to the Medico-Chirurgical Hospital; Lecturer on Medical

Terminology and Symptomatology, and Demonstrator of Physleal Dlagnosis in the Medico-Chirurgical College. PHILADELPHIA.

The importance of Roentgen examinations of the lungs is well expressed by the statement of Dr. Judson A. Daland, who says: "No chest examination is complete without an $x$-ray examination." In order to make Roentgen examinations of the greatest possible value, the best technic and the most careful and accurate interpretation must be employed.

As specialists in this special branch of medicine, we have made great strides in the perfection of our technic, but even with the most perfect technic in the making of a negative, much general knowledge of medicine, and much experience is still needed to interpret accurately the shadows obtained. Much assistance can be obtained from the study of the literature, but much more can be learned by the actual study of the negatives of the various pathologic conditions, associated wtth the clinical or the postmortem findings.

Postmortem examinations are seldom made immediately after the Roentgen examination, and in many of our cases they are never made. No one of us, therefore, has seen explanations of all the shadows obtained in our negatives. Neither has any one of us had the opportunity of studying all the pathologic conditions that we may be called on to examine. I have decided, therefore, to present before this body a number of Roentgenograms, showing a variety of pathologic conditions of the lungs, and to give my interpretation. In many instances, I know that my interpretation is correct, but in others, some of my colleagues may differ with me. It is with the hope of bringing out criticisms and thus of combining our experience in the study of diseases of the lungs, that I present this subject.

Fluoroscopic examinations of the lungs have been made almost since the discovery of the Roentgen rays, but the actual progress in the Roentgen diagnosis of diseases of the lungs dates from the beginning of short exposures. In this field excellent work has been done by Hulst, Rieder and others.

In order to study the lungs accurately, the negative must be made while the lungs are at rest, or, in other words, while the patient holds the breath. This will usually be from three to fifteen seconds. I have succeeded in-making several good negatives of the chest of a child of 9 years of age in one-tenth of a second. Such short exposures are not necessary in the study of the lungs, but are necessary for the accurate study of the heart.

* Read at the Annual Meeting of the American Roentgen Ray Society, Baltimore, Sept. 28-30, 1905.
As a rule, a careful physical examination should precede the Roentgen examination, not because it will modify the shadows, but because it will enable the Roentgenologist to interpret those present, and may modify the method used, and thus make a second examination unnecessary. The greatest field of usefulness of the Roentgen rays in lung diseases is the study of tuberculosis. Here the lesions can be recognized earlier and more accurately than by any other method. Besides assisting in making a diagnosis, it is the most valuable and the most accurate method of recording the lesions at the various stages of the disease, and thus enables the physician to estimate the value of his therapeutic procedures.

METHOD OF MAKING THE EXAMINATIONS.

I have practically discontinued the use of the fluoroscope, because of its inaccuracy and because of the dangers attending its use. By the use of the modern devices for the protection of the operator at least one of these objections can be removed; still, I feel that a fluoroscopic examination is not necessary. Generally, a negative should be made with the plate posterior to the patient and another with the plate anterior to the patient, so as to give proper value to any lesions lying near to the surface, and in ordei to recognize any thickening of the pleura. As a rule, the patient should be placed in the recumbent posture, except when pleural effusion is suspected, in which case the patient should be examined in the sitting posture. The tube should be placed at a distance of from 18 to 20 inches from the plate. The time of the exposure will vary with the time that the patient can hold the breath, and this time should be accurately determined by testing the patient several times before attempting to make the exposure.

\section{INTERPRETATION.}

The proper interpretation of the negative is more important than the making of it and fully as difficult. The negative itself should be used for study.

Old calcified tubercles give the most decided shadows, and lesions of this kind can be recognized as small as one-eighth of an inch in diameter. Old scars or fibrous tissue cast a less dense shadow and require larger lesions. but they can usually be recognized by their band-like appearances.

Consolidations vary in the density of their shadows with the size of the lesion; but an area one-half inch in diameter can be recognized in an emaciated person. In one patient, who was very much emaciated as the result of tuberculosis of the peritoneum, I succeeded in showing little tubercles the size of a pin head. Consolidations seldom occur singly, therefore, they give the lung a mottled appearance, except when there is massive consolidation. Finally, the consolidation can be recognized by comparison of the suspected area with that of the opposite lung or with the other parts of the same lung. The tubercular deposits, or the affected areas lying in different planes, are thrown on the plate in one plane; therefore, on superficial observation, one might conclude that a greater proportion of the lung is affected than is correct. That is, the whole lung might show a mottled appearance, and the false conclusion be drawn that no healthy lung tissue remained. This mistake need not be made by an experienced observer, because if the whole lung or lobe is involved the degree of general density will be much greater than when only scattered areas are involved, and the density will approach that of the shadow of the heart. Even when a whole lobe is involved, the shadow is rarely uniformly dense, because the 